\title{
LA MESURE D'UN RAYONNEMENT GAMMA PAR UNE CHAINE DE DETECTION
}

\author{
J. JACOT * \\ (Manuscrit reçu le 24 juin 1968 )
}

\begin{abstract}
RÉSUMÉ
Après avoir analysé les processus de la détection d'un rayonnement gamma par un appareil de mesure et donné la forme des informations obtenues à la sortie de l'appareil, on établit les diverses étapes permettant, à partir d'un rayonnement d'activité donnée de prévoir la valeur du phénomène détecté puis, une fois bien établi l'étalonnage de la chaîne de détection, on décrit les méthodes de dépouillement des spectres d'impulsion d'un rayonnement polyénergétique discret et d'un rayonnement continu.
\end{abstract}

\section{INTRODUCTION}

Pour rattacher les résultats théoriques d'un calcul aux résultats de l'expérience qui mesure un phénomène, il importe de faire une étude précise de la réponse de l'appareil de mesure à ce phénomène. Il y a donc une correspondance à établir entre le flux de rayonnement entrant et les résultats des mesures enregistrées.

Le problème ne peut pas se traiter directement : il faut le décomposer et c'est cela que nous ferons dans une première partie. Mais si cette façon de procéder est relativement simple, le problème de connaître, à partir des résultats de mesure, le phénomène responsable est autrement compliqué. Pourtant il est indispensable d'étudier le transfert dans les deux sens; connaissant l'un on en déduit l'autre en inversant les phénomènes.

Pour illustrer l'aspect de la réponse d'un appareil de mesure nous allons décrire la détection d'un rayonnement $\gamma$ par les cristaux inorganiques scintillants.

\section{I - CHAINE DE DÉTECTION DU RAYONNEMENT GAMMA}

\section{PROCESSUS DE DÉTECTION}

Un rayonnement $\gamma$ émis par la source I subit dans le détecteur 2 des interactions et l'énergie perdue lors de ces interactions est convertie en énergie lumineuse à l'aide d'un dopant (atome de Tl dans le $\mathrm{Na} \mathrm{I}$ ). Cette énergie lumineuse,

* Attaché aux Services techniques des Armées, Laboratoire Central de l'Armement 16 bis, avenue Prieur-de-la-Côte-d'Or, 94 - Arcueil. 
par effet sur la photocathode sensible du P.M. 3 se convertit en impulsions électriques qui sont amplifiées en 4 puis sélectionnées par un sélecteur d'amplitude multicanaux 5 . Il y a alors numérisation de l'impulsion, visualisée en 6 soit sur écran oscilloscopique soit par sortie numérique.

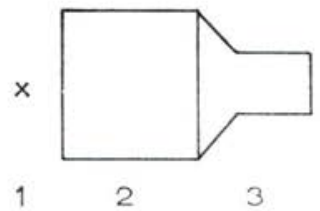

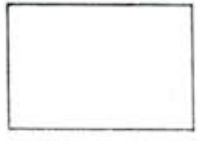

4

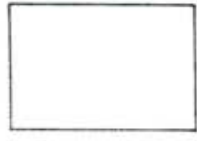

5

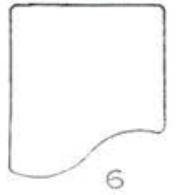

6

Chaine de détection.

I - Source émettrice de rayonnement. - 2 - Détecteur-cristal inorganique. - 3 - Photomultiplicateur d'amplitude. - 5 - Sélecteur d'amplitude. - 6 - Sortie des résultats.

C'est le transfert d'énergie que l'on va étudier pas à pas car il est impossible d'en faire un bilan global et à fortiori, à partir des informations recueillies remonter au phénomène responsable.

\section{I - LA SOURCE}

Que ce soit une source ponctuelle, une source plane à l'infini ou à distance finie, ou une source volumique, il est nécessaire de bien connaître dans quelle direction et avec quelle intensité elle émet.

L'hypothèse de la source ponctuelle ne peut être retenue quand on connaît la forme des sources utilisées pour les expériences. En effet ces sources sont soit étendues sur un support, soit contenues dans un récipient; l'absorption du support ou des parois du récipient influe sur l'activité propre de la source c'està-dire qu'il y a atténuation du flux de rayonnement à la sortie de la source; d'autre part dans le cas de solutions, l'absorption du solvant n'est pas négligeable.

On peut écrire la relation qui existe entre l'activité propre de la source et l'activité réellement mesurée par le détecteur.

$$
I_{\text {exp }}=I_{o} f\left(\mu_{o}, \mu_{p}, \overrightarrow{r_{o}}, \overrightarrow{r_{p}}\right)
$$

où $\mu_{0}$ et $\mu_{p}$ sont les coefficients d'absorption du solvant et des parois

et $\overrightarrow{r_{o}}$ et $\overrightarrow{r_{p}}$ représentent les épaisseurs de matière traversée dans la direction vers laquelle s'effectue la mesure.

Pour déterminer ce phénomène, on peut réaliser l'expérience suivante :

La source est diluée dans de l'eau contenue dans un tube à essais; en faisant varier le volume d'eau, c'est-à-dire la hauteur de la source, et pour un même temps de comptage (pour un émetteur de période longue) on établit le tableau I. 
TABLEAU I

\begin{tabular}{|c|c|c|c|c|c|c|}
\hline \multirow{2}{*}{ 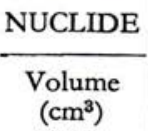 } & \multicolumn{2}{|c|}{${ }^{51} \mathrm{Cr} \quad \mathrm{N}_{e}=1.003716$} & \multicolumn{2}{|c|}{${ }^{137} \mathrm{Cs} \quad N_{e}=885.600$} & \multicolumn{2}{|c|}{${ }^{65} \mathrm{Zn} \quad N_{e}=622.350$} \\
\hline & $N_{c}$ & $N_{c} / N_{e}$ & $N_{c}$ & $N_{c} / N_{e}$ & $N_{c}$ & $N_{c} / N_{e}$ \\
\hline I & 870120 & 0.870 & 367628 & 0.415 & 152209 & 0.244 \\
\hline 3 & 866317 & 0.866 & 368436 & 0.416 & I 2289 & 0.244 \\
\hline 5 & $8 6 \longdiv { 3 9 8 }$ & 0.865 & 372009 & 0.420 & 149965 & 0.240 \\
\hline 7 & 862722 & 0.862 & $368 \circ 48$ & 0.415 & 144899 & 0.232 \\
\hline Io & 846807 & 0.846 & 366492 & 0.413 & 142620 & 0.229 \\
\hline 13 & 830872 & 0.830 & 363423 & 0.410 & 140089 & 0.225 \\
\hline 16 & 808925 & 0.802 & 355938 & $0.40 \mathrm{r}$ & 136172 & 0.218 \\
\hline
\end{tabular}

$N_{e}=$ Nbre de photons émis par la source

$N_{c}=$ Nbre d'événements comptés.

L'établissement de la formule permettant le calcul de l'auto-absorption de la source cylindrique donne la valeur du nombre de photons absorbés.

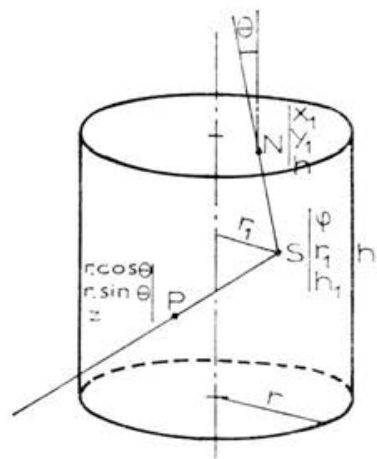

Déjà, dans ce cas simple, cela fait appel à un calcul compliqué :

Le nombre de photons s'échappant par la face parallèle est fourni par

$$
N_{1}=2 \pi \int_{0}^{r} r_{1} \mathrm{~d} r_{1} \int_{0}^{b}\left(b-b_{1}\right) \mathrm{d} h_{1} \int_{0}^{2 \pi} \int_{0}^{r} \frac{\mathrm{e}^{-\mu \mathrm{d}(\rho, \theta)}}{\mathrm{d}(\rho, \theta)^{3}} \rho \mathrm{d} \rho \mathrm{d} \theta
$$

et le nombre $\mathrm{N}_{2}$ de photons s'échappant par la surface latérale par

$$
N_{2}=2 \pi r \int_{0}^{r} r_{1} \mathrm{~d} r_{1} \int_{0}^{b} \mathrm{~d} z \int_{0}^{2 \pi} \int_{0}^{b} \frac{\mathrm{e}^{-\mu \mathrm{d}(\theta, z)}\left(r-r_{1} \sin \theta\right) \mathrm{d} z \mathrm{~d} \theta}{\left[r^{2}+r_{1}^{2}+\left(z-b_{1}\right)^{2}-2 r r_{1}-\sin \theta\right]^{3}} .
$$


Le nombre de photons émis par la source est :

$$
N_{T}=4 \pi \mathrm{V}=4 \pi^{2} r^{2} b .
$$

Le facteur d'auto-absorption s'écrit :

$$
\mathrm{R}=\frac{N_{T}-N_{2}-2 N_{1}}{N_{T}}
$$

La comparaison des résultats expérimentaux et des résultats du calcul se fait alors pour des énergies bien déterminées; l'interpolation est alors possible. La formule ( $\mathrm{I}$ ) si elle est en soi valable, néglige certainement des effets du rayonnement dans le milieu : rétrodiffusion des $\gamma$ sur les parois, perte partielle d'énergie, etc. Aussi, pour reproduire la réalité, faudra-t-il apporter quelques corrections dans les données géométriques.

Voilà donc déterminé le flux $\gamma$ susceptible d'être détecté et mesuré.

\section{2 - LA GÉOMÉTRIE SOURCE-DÉTECTEUR}

Si l'on suppose la source émettant dans tout l'espace et un détecteur situé à une distance d de celle-ci, seuls les rayons $\gamma$ émis dans un angle solide :

$$
\Omega=2 \pi(\cos \theta-\mathrm{I}) \quad \text { ou } \quad \Omega=2 \pi \frac{r}{\sqrt{\mathrm{d}^{2}+r^{2}}}(2) \quad(r=\text { rayon du détecteur })
$$

seront détectés. On appelle facteur de géométrie $G$ le rapport du nombre de $\gamma$ détectés au nombre de photons détectables.

$$
G=\frac{\Omega}{4 \pi} \quad(4 \pi=\text { angle solide de tout l'espace }) .
$$

La détermination de $\Omega$ présente quelques difficultés sauf dans le cas d'une source ponctuelle car dans ce cas la formule (2) est aisée à calculer.

Lorsque la source est sous la forme d'un disque ou sous la forme d'un cylindre ou d'une sphère, on assimile la source à une multitude de micro-sources, de densité d'activité unité par élément de surface ou de volume et on intègre ensuite.

Le calcul de $\Omega$ peut faire appel à des techniques élaborées comme la méthode de Monte-Carlo [ $\mathrm{I}$ ], ou au calcul d'intégrales multiples.

Pourtant il est généralement plus simple de faire un calcul global de l'angle solide, de l'absorption de la source et de l'efficacité du détecteur.

Du point de vue expérimental on remarque qu'une bonne détection est fonction de $G$; on se rapproche de la valeur maximum $G=\mathrm{I}$ de deux manières (détection $4 \pi$ ) :

- cristal puits (fig. I),

- cristaux plats en coïncidence (fig. 2). 


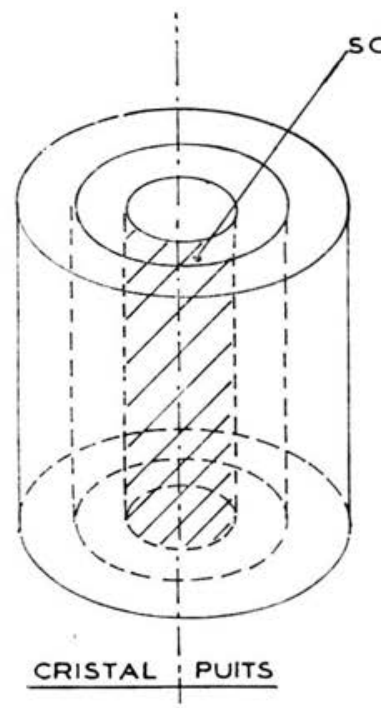

FIG. I

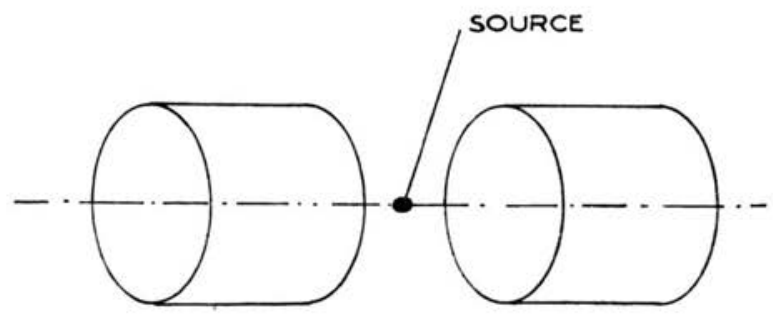

CRISTAUX PLEINS EN COINNCIDENCE

FIG. 2

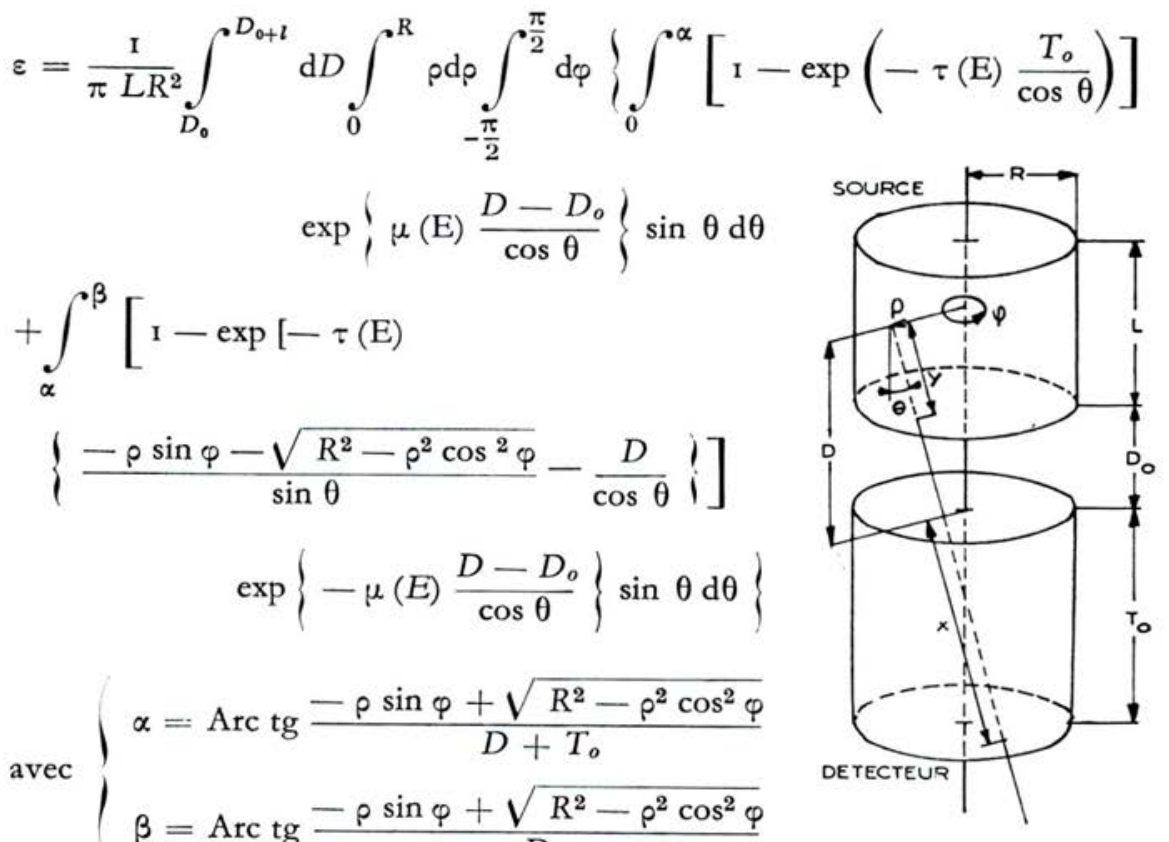

FIG. 3 
I.3 - L'EFFICACITÉ DU DÉTECTEUR

Ayant choisi pour notre exposé un détecteur à scintillation (cristal de $\mathrm{Na} \mathrm{I}$ (T l) ) on définit l'efficacité comme le rapport du nombre de photons détectés au nombre de photons atteignant le cristal.

$$
\varepsilon_{i}=\frac{N_{d}}{N_{a}}
$$

Pour rattacher le résultat de mesure à l'activité de la source, il faut faire intervenir le facteur de géométrie et on définit alors l'efficacité absolue par :

$$
\varepsilon_{a}=\frac{\varepsilon_{i}}{\bar{C}_{\mathrm{r}}} \cdot \mathrm{R} \text {. }
$$

Ces deux paramètres sont fonction de l'énergie et des dimensions du cristal détecteur. Il s'agit de déterminer l'absorption des $\gamma$ dans le cristal.

La figure 3 représente un dispositif courant de mesure. La source a un rayon égal au cristal ce qui est réalisable en laboratoire; mais il est loisible de faire varier ce rayon et la distance de la source au cristal.

La formule générale de $\varepsilon$ est

$$
\varepsilon=\frac{\int[1-\exp (-\mu(E) \cdot x] \exp -(\sigma(E) \cdot z) \mathrm{d} \Omega}{\int_{\Omega} \mathrm{d} \Omega} .
$$

Pour montrer la complexité d'un calcul d'efficacité dans le cas choisi, nous donnons la formule utilisée au dispositif de la figure 3 .

$\mu$ et $\tau$ étant le coefficient d'absorption dans la source et dans le cristal.

La figure 4 donne l'allure de l'efficacité pour cette géométrie. On y remarque l'influence de l'auto-absorption aux basses énergies et le léger décalage par la suite.

Des calculs semblables ont été effectués par SNYDER [2] et au L.C.A. [3] pour des détecteurs puits. La comparaison des résultats de mesure du même genre que ceux rapportés dans le tableau I et des calculs est satisfaisante.

Pourtant on ne saurait négliger l'enveloppe du cristal, mince couche métallique à fort pouvoir réfléchissant de radiations U.V. Cette enveloppe contribue à l'atténuation des $\gamma$ à basse énergie (fig. 5).

En dehors d'une absorption du rayonnement, il se produit dans le détecteur une dégradation d'énergie provenant de l'interaction des photons avec la matière.

Un photon $\gamma$ subit dans un milieu trois grands types d'interaction :

- effet photoélectrique,

- diffusion Compton,

- effet de paires. 
RAYON COMMUN: $3.81 \mathrm{~cm}$

EPAISSEUR CRISTAL: $7.62 \mathrm{~cm}$

EPAISSEUR SOURCE: $1.30 \mathrm{~cm}$

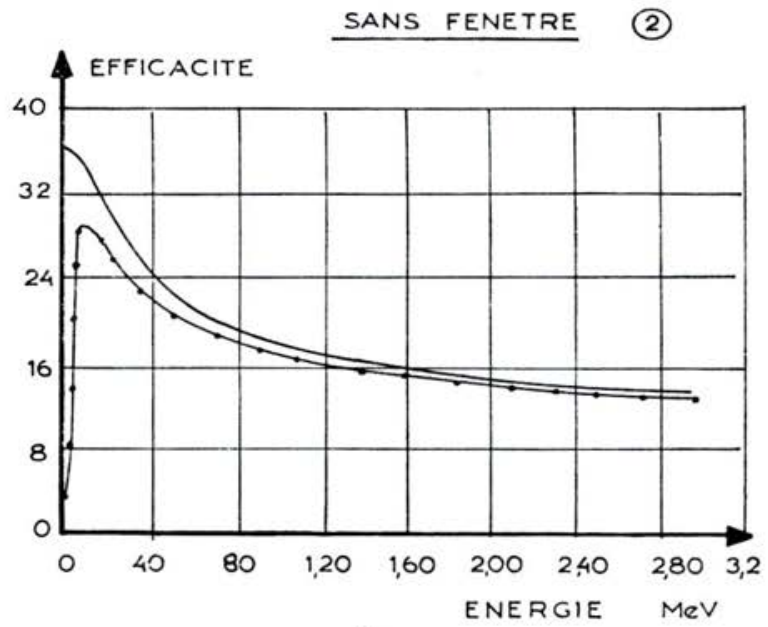

FIG. 4

SANS AUTO ABSOREY!'IN

AVEC AUTO ABSORPTION

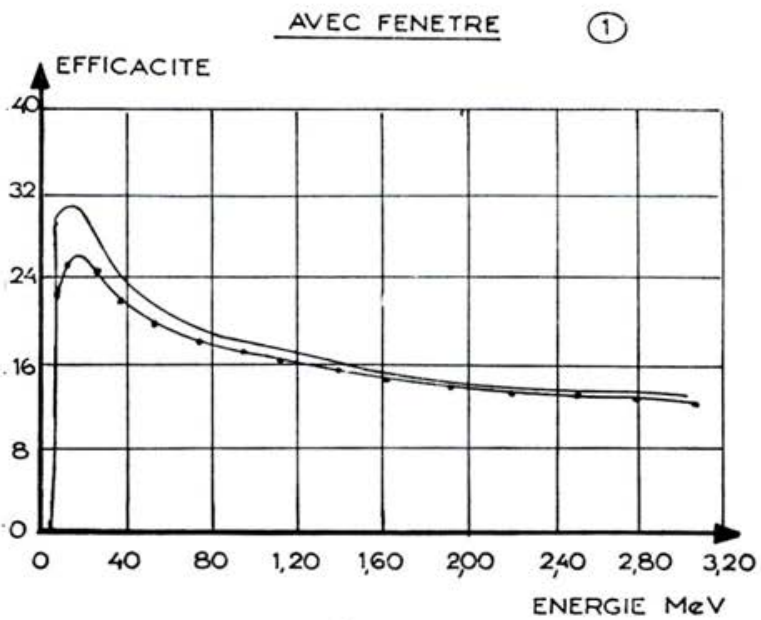

FIG. S 
Nous rappelons les divers effets :

\section{I) Effet photoélectrique}

Le photon subissant un effet photoélectrique cède son énergie à un électron du cristal. Sans entrer dans les détails, on peut dire que tout photon ayant un effet photoélectrique est absorbé et son énergie provoque une excitation des atomes du dopant qui réémet dans l'U.V.

\section{2) Diffusion Compton}

Par ce processus le photon cède son énergie pour partie à un électron, et pour partie à une réémission d'un autre photon d'énergie plus faible.

L'énergie électronique peut être considérée comme absorbée; quant au photon réémis, il subit des interactions semblables au photon primaire. Néanmoins il peut s'échapper du cristal et dans ce cas on voit immédiatement que du photon primaire seule une partie de son énergie est captée. Cela donne une distribution continue d'énergie de $O$ à une valeur fixée $E$ par rapport à l'énergie initiale $E_{0}$;

$$
E=\frac{2 E_{0}^{2}}{2 E_{0}+5 \mathrm{II}}(\text { exprimé en } \mathrm{keV}) \text {. }
$$

\section{3) Effet de matérialisation}

Pour une énergie supérieure à $1,022 \mathrm{MeV}$, au voisinage du noyau, un $\gamma$ peut donner naissance à une paire positon-électron. Cette matérialisation, d'électron qui peut être absorbé, et de positon qui avec un électron du cristal donne naissance à deux photons d'énergie fixée à 0,5 I I MeV, conduit à la formation de deux raies d'échappement si l'un ou l'autre photon ou les deux quittent le cristal sans interaction. La distribution d'énergie dans le détecteur peut être représentée par la figure 6 .

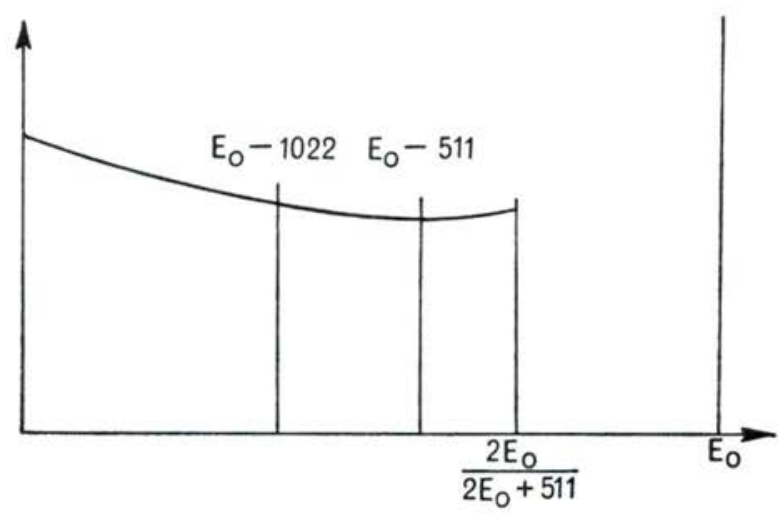

Frg. 6. - Spectre d'électrons à la sortie du détecteur. 
Ceci figure le spectre pour un rayonnement monoénergétique $E_{0}$. La distribution Compton peut être obtenue par la formule de KLEIN-Nishina.

\section{I.4 - LA CONVERSION ET LA MULTIPLICATION DES IMPULSIONS}

L'énergie lumineuse résultant de l'excitation des atomes du dopant est recueillie sur la photocathode d'un photomultiplicateur sensible au rayonnement U.V. Par un système de dynode représenté figure 7 , il se produit une multiplication électronique et un courant notable est recueilli sous forme d'impulsions. On peut dire que la détection d'un photon $\gamma$ est responsable d'une impulsion à la sortie du P.M., dont la hauteur est proportionnelle à l'énergie absorbée par le détecteur (fig. 8).

L'analyse finale des trains d'impulsions se fait par un sélecteur d'amplitude qui partage l'amplitude de l'impulsion et la distribue dans des canaux de largeur fixée $\Delta \mathrm{V}$; cette largeur s'appelle le gain du sélecteur et s'exprime en $\mathrm{keV} / \mathrm{canal}$, ceci pour faire le lien entre l'énergie du photon analysé et la position de l'impulsion correspondante. Chaque impulsion d'amplitude représente un coup dans le canal; il n'est donc pas possible de classifier très nettement l'énergie, et on parle de valeur moyenne d'énergie dans un canal.

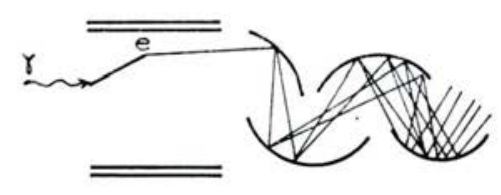

FIG. 7. - Système de dynodes.

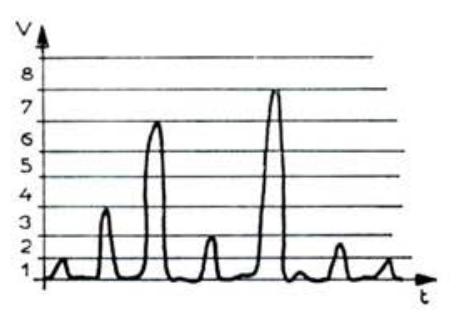

FIg. 8. - Train d'impulsions.

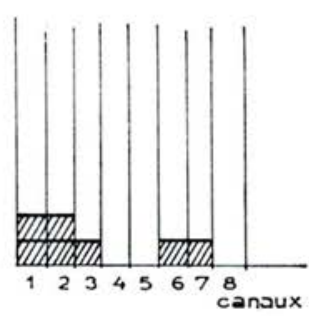

Fig. 9. - Classement des impulsions-sélecteur.

Comme il s'agit d'un comptage, d'une conversion d'énergie électroniquelumineuse et électrique il y a multiplication du phénomène.

Il se produit donc une certaine dispersion et on constate effectivement au niveau de la raie d'absorption totale, une distribution d'allure gaussienne centrée sur cette raie.

\section{S - LE SPECTRE FINAL}

Reprenant la figure 6, la statistique de comptage, la photomultiplication et la dispersion lumineuse, un spectre $\gamma$ se présente sous la forme donnée par la figure ro.

En fait, à la sortie du sélecteur, c'est sous la forme d'un histogramme (fig. I I) que sort le spectre. Comment alors exprimer cette observation expérimentale à partir de la distribution énergétique à mesurer ? 


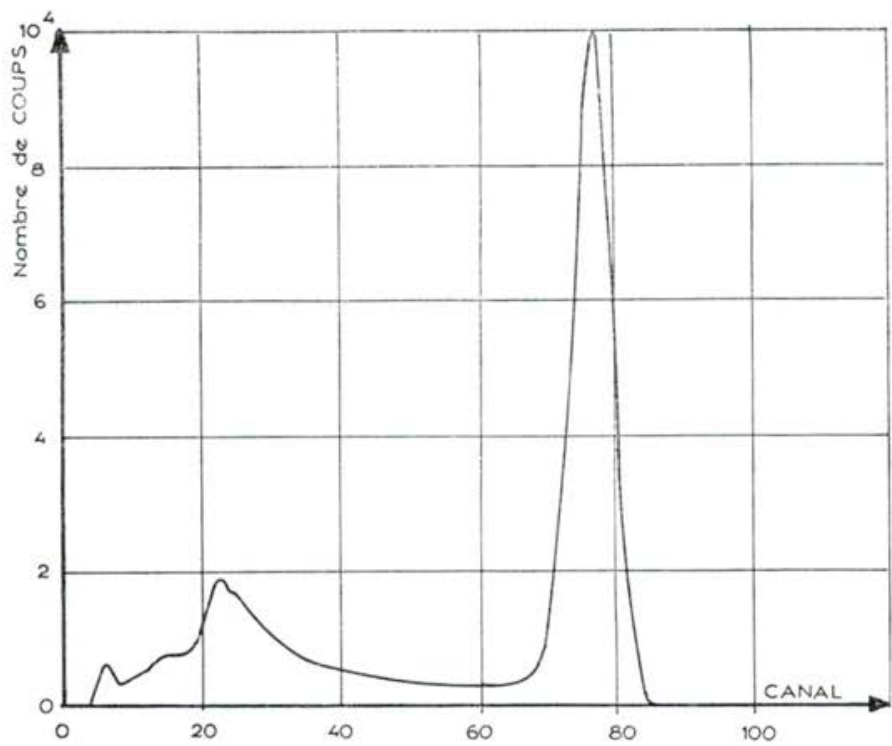

FIG. I0. - Réponse d'un détecteur à un rayonnement monoénergétique.

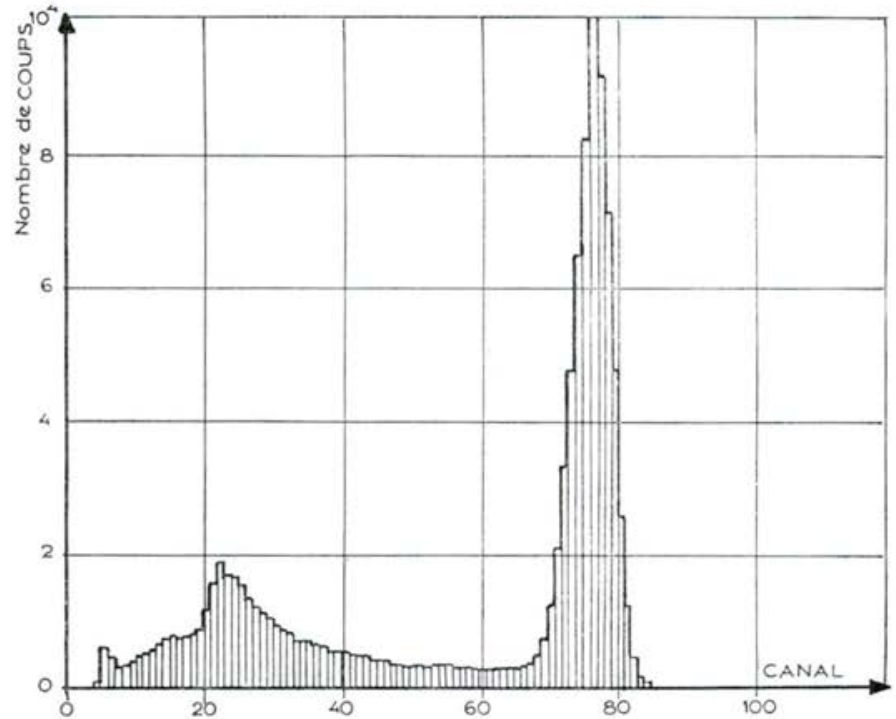

FIG. II. - Histogramme du spectre d'impulsion. 
Dans le cas simple d'un rayonnement monoénergétique soit $F(E)$ la distribution spectrale est donnée par :

$$
F\left(E_{0}\right)=\int_{0}^{\infty} \alpha_{0} \delta\left(E-E_{0}\right) f(E) \mathrm{d} E
$$

où $\alpha_{0}$ est l'intensité du rayonnement d'énergie $E_{0}$ reliée à l'activité de la source par $\alpha_{0}=A \times \tau(\tau=$ taux de comptage $)$ et $\delta\left(E-E_{0}\right)$ la distribution de Dirac.

Dans le détecteur, par suite des absorptions diverses vues plus haut : atténuation sur les parois de la source, dans la source, sur l'enveloppe du cristal et dans le cristal,

$$
F\left(E_{0}\right)=\varepsilon\left(E_{0}\right) \times R(E .) \int_{0}^{\infty} \alpha_{0} \delta\left(E-E_{0}\right) f(E) \mathrm{d} E .
$$

La réponse du détecteur donne le spectre d'impulsion $F(V)$ en amplitude

$$
F(V)=\int_{0}^{\infty} F\left(E_{0}\right) K\left(V, E_{0}\right) \mathrm{d} E_{0} .
$$

$K\left(V, E_{0}\right)$ exprimant la réponse du détecteur (fig. 9, ro et i 1 ). Mais l'information utilisable pour les calculs est donnée en nombre de coups par canal de l'histogramme des amplitudes. Et si $F(V)$ a l'allure du spectre donné figure ro, la quantité $N_{i}$ désignant le contenu du canal numéro $i$ du spectre de la figure in, est reliée à $F(V)$ par

$$
N_{i}=\int_{\Delta V_{i}} F(V) \mathrm{d} V
$$

C'est donc à partir de ces informations élémentaires qu'il convient de chercher la réponse du détecteur et sa capacité à reproduire les événements qu'on lui demande de compter.

\section{DÉTERMINATION DE $K(V, E)$}

Brièvement, disons que l'on a souvent recherché à déterminer $K(V, E)$ par une expression analytique. On observe tout d'abord qu'il est aisé de représenter le pic d'absorption totale par une gaussienne :

$$
g(V, E)=a \exp \left\{-\left(\frac{V-E}{b(E)}\right)^{2}\right\}
$$

$a=$ facteur de normalisation,

$b$ = écart-type dépendant de l'énergie, 
Déjà pour cette partie du spectre l'accord avec les résultats de l'expérience divergent. Heath [4] propose :

$$
g(V, E)=a \exp \left\{-\left(\frac{V-E}{b(E)}\right)^{2}\left\{\left(\mathrm{r}+\beta_{1}(V-E)^{n}+\beta_{2}(V-E)^{r}\right)\right.\right.
$$

$\beta_{1}, \beta_{2}, r$ et $n$ d'une part et $a, E, b$ sont des paramètres fixés pour une énergie et une chaîne de détection données.

Ajuster un pic, compte tenu de la relation (10) devient réellement fastidieux. En se contentant de l'expression (I I ) on parvient aisément à déterminer $a, b$ et $E$ caractérisant respectivement les hauteurs, l'écart-type relié à la largeur à mi-hauteur et la position du pic d'absorption totale et même des pics de simple et double échappement.

Pour le fond Compton - partie continue du spectre - Heath lisse avec un polynôme.

$$
b(V, E)=c+\mathrm{d} V+\sum_{h=1}^{N h} b_{h} \sin \frac{b \pi V}{L}
$$

où $L$ est la largeur des bandes dans lesquelles l'accord entre la série et la distribution spectrale doit se faire.

La représentation analytique d'un spectre d'amplitude gamma monoénergétique s'écrit alors :

$$
K(V, E)=g(V, E)+g_{s}(V, E)+g_{d}(V, E)+b(V, E)
$$

en n'omettant pas que l'ajustement des paramètres - tous dépendant de l'énergie du nuclide émetteur - doit se faire en accord avec la relation (ro).

Donnons encore une autre expression du fond Compton, due celle-ci à Chester [ 5 ].

$$
\begin{aligned}
b(V, E)=\gamma_{1}\left[\frac{\mathrm{I}+\gamma_{2}{ }^{2} \exp \left(\frac{V-\gamma_{3}}{\gamma_{4}}\right)}{\exp \left(\frac{V-\gamma_{3}}{\gamma_{4}}\right)+\left[\mathrm{I}-\gamma_{2} \exp \left\{-\left(\frac{V-\gamma_{3}}{\gamma_{4}}\right)^{2}\right\}\right]}\right]^{1 / 2} \\
+\gamma_{5}\left(\frac{V-\gamma_{6}}{\gamma_{7}}\right) \exp \left[\left(\frac{V-\gamma_{6}}{\gamma_{7}}\right)-\mathrm{I}\right] \cdot \\
\text { II - LA DÉTECTION } \\
\text { D'UN RAYONNEMENT POLYÉNERGÉTIQUE }
\end{aligned}
$$

De la même façon qu'on a défini un rayonnement monoénergétique par (6) un rayonnement polyénergétique peut s'écrire :

$$
F(E)=\sum_{h} \int_{0}^{\infty} \alpha_{h} \delta\left(E-E_{h}\right) f(E) \mathrm{d} E .
$$

Expérimentalement le spectre représentatif est la source pondérée par $\alpha_{h}$ de rayonnements monoénergétiques d'activité $\mathrm{A}$. 
La figure 12 représente un tel spectre somme de deux rayonnements.

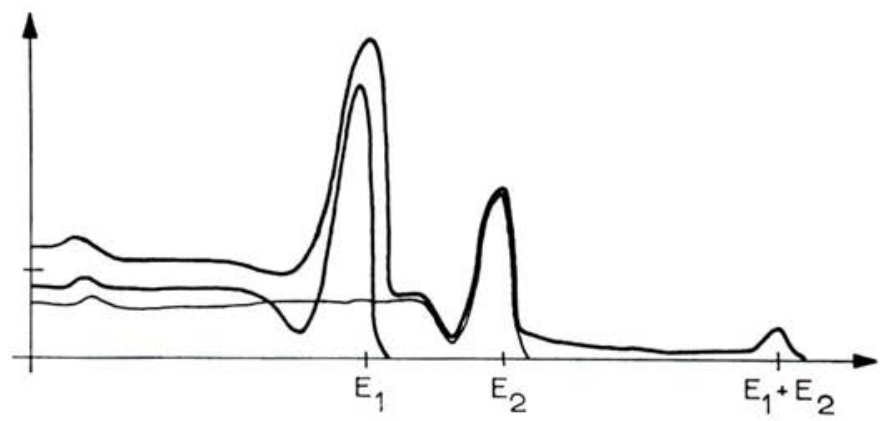

FIG. I 2.

On n'observe pas tout à fait un spectre somme; en fait, à cause du temps mort de la chaîne de détection il peut se produire des coïncidences de photons et le détecteur réagira comme à un rayonnement d'énergie somme des deux énergies. C'est le cas fréquent des cascades $-{ }^{60} \mathrm{Co}$. Un pic somme se situera à l'amplitude correspondant à la somme des amplitudes des rayonnements, avec en plus toutes les énergies intermédiaires dues aux pertes partielles du rayonnement dans le cristal.

Une évaluation quantitative du pic somme est difficile et il est évident qu'il perturbe les pics d'absorption totale de chacun des deux émetteurs.

\section{I - LA CARACTÉRISTISATION DES ÉNERGIES}

Les constituants d'un spectre polyénergétique sont caractérisés par la position de chacun des pics d'absorption totale apparents. En ajustant au mieux une gaussienne à ces pics on détermine aisément les paramètres caractéristiques du rayonnement. Donnons sommairement la marche à suivre.

Soit l'équation de la gaussienne :

$$
y=a \exp \left\{-\left(\frac{E-V}{b}\right)^{2}\right\}
$$

et $S_{i}=\int_{\Delta V_{i}} y \mathrm{~d} V$ la contribution de la gaussienne dans le canal numéro $i$ du sélecteur d'amplitude.

Si $N_{i}$ est le nombre de coups effectivement relevé dans le canal $i$ au cours de l'expérience, l'erreur statistique de comptage s'écrit :

$$
R=S_{i}-N_{i} \text {. }
$$

En minimisant la somme des carrés des erreurs dans l'intervalle de bonne définition du pic par rapport aux trois paramètres $a, b$ et $E$. 
soit $\frac{\partial \sum_{i} R_{i}{ }^{2}}{\partial a}=0 ; \frac{\partial \sum_{i} R_{i}{ }^{2}}{\partial b}=0 ;$ et $\frac{\partial \sum_{i} R_{i}{ }^{2}}{\partial E}=0$,

on ajuste au mieux le pic par la gaussienne la plus probable.

Aussi pour tous les pics visibles d'un spectre polyénergétique, on peut déterminer les amplitudes des pics et si auparavant on a étalonné le spectromètre, c'est-à-dire relevé pour une série de spectres monoénergétiques témoins la correspondance - amplitude - énergie, on en déduit aisément les énergies des corps entrant dans la composition du spectre complexe.

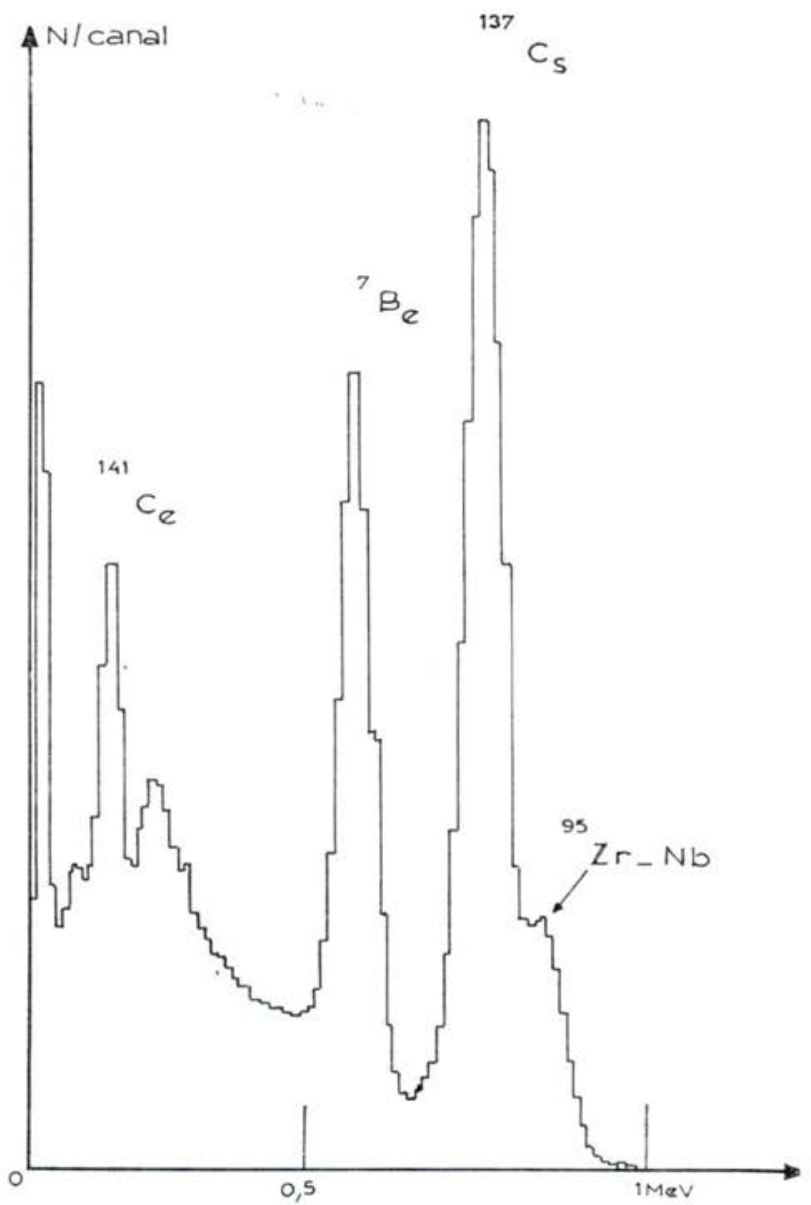

FIG. 13. - Spectre de produits de fission.

La figure I 3 donne une représentation d'un tel spectre. A partir de la figure I4 donnant la courbe d'étalonnage du spectromètre utilisé on a porté les corps susceptibles d'être présents dans ce mélange. 
II. 2 - LA RÉPONSE DU DÉTECTEUR. LA SPECTROTHÈQUE

Avant tout dépouillement il importe de constituer une spectrothèque de corps que l'on peut rencontrer fréquemment dans les mélanges à dépouiller. Ceci nécessite le relevé de nombreux spectres et un calage parfait de l'appareillage; en effet il faut que la base sur laquelle sont relevés les spectres dits étalons soit la même. La température, la tension et même la stabilisation automatique influent d'une façon certaine sur le calage. Par un programme mis au point au L.C.A.- RECAL - la correction de dérive et de gain est apportée à chaque spectre.

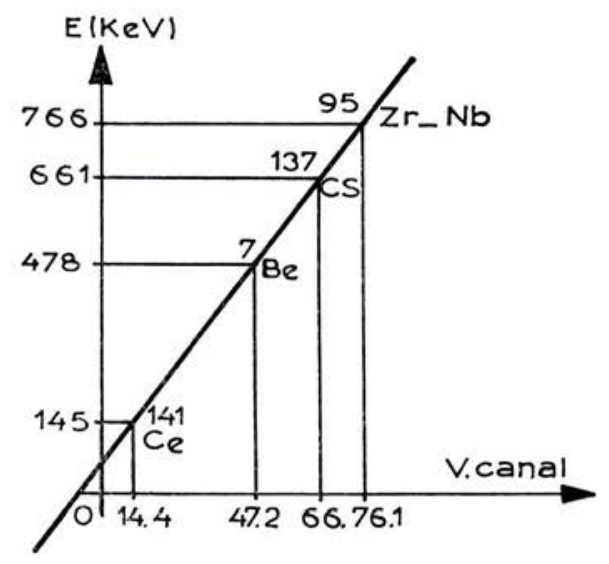

Fig. 14. - Courbe d'étalonnage d'un spectromètre.

Si cette correction n'a pas d'importance notable dans le cas de spectres simples de quelques éléments, elle est absolument nécessaire pour un mélange très complexe, à savoir constitué de pics qui se chevauchent et dont il est impossible de discerner leur position exacte.

\section{3 - LE DÉPOUILLEMENT}

Un mélange de spectres est considéré comme une source pondérée de spectres étalons : dans un canal $i$ le nombre de coups relevés $b_{i}$ est combinaison linéaire du nombre de coups $a_{i j}$ dans ce canal des spectres étalons $j$.

$$
b_{i}=\sum_{j} a_{i j} x_{j}
$$

$x_{j}=$ proportion de l'étalon $j$ entrant dans le complexe.

Si l'on avait affaire à un comptage fidèle sans erreurs statistiques il est évident que pour dépouiller un spectre composé de $n$ corps la valeur du nombre de coups dans $n$ canaux suffirait. 
En effet :

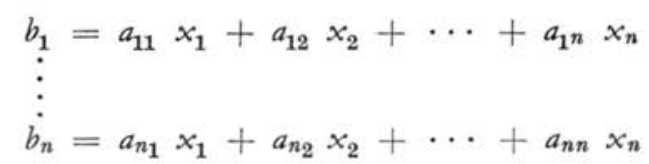

soit $B=A X(\mathrm{r} 4)$ en notation matricielle

$B$ vecteur de composantes $\left(b_{1} \cdots b_{n}\right)$

$X$ vecteur solution $\left(x_{1} \cdots x_{m}\right)$

et $A$ matrice d'éléments $a_{i j}$.

En résolvant le système $B-A X=0$ ou en inversant $A$ soit $X=A^{-1} B$ on en tire la valeur de $X$.

Ceci n'est pas le cas lorsqu'il s'agit de comptage. En introduisant l'erreur $Z_{i}$ sur le comptage du spectre à analyser, la relation (1 2) devient :

$$
b_{i}=\sum_{j} a_{i j} x_{j}+Z_{i}
$$

(cela sous-entend qu'il n'y a pas d'erreur statistique sur le comptage de spectres étalons).

La méthode des moindres carrés revient à minimiser la somme des carrés des erreurs soit $\mathrm{R}=\sum_{i} Z_{i}{ }^{2}$ par rapport aux $x_{h}$ :

$$
\frac{\partial \mathrm{R}}{\partial x_{h}}=0 \cdot \forall b .
$$

Détaillons le calcul :

$$
\begin{gathered}
\frac{\partial \mathrm{R}}{\partial x_{h}}=2 \sum_{i} Z_{i} \frac{\partial Z_{i}}{\partial x b}=0 \\
Z_{i}=\left(b_{i}-\Sigma_{j} a_{i j} x_{j}\right) \quad \text { et } \quad \frac{\partial Z_{i}}{\partial x_{h}}=-a_{i h}
\end{gathered}
$$

on obtient

$$
\sum_{i} b_{i} a_{i h}=\sum_{j} \sum_{i} a_{i h} a_{i j} x_{j}
$$

en posant

$$
B_{h}=\sum_{i} b_{i} a_{i h}
$$

et

$$
\begin{aligned}
& A_{j h}=\sum_{i} a_{i h} a_{i j} \\
& B_{h}=\sum_{j} A_{j h} x_{j}
\end{aligned}
$$

relation du type de la formule (13),

ou

$$
B=A X \text {. }
$$


RÉSOLUTION DU SYSTÈME (I6)

L'inversion d'une matrice sur ordinateur pose des problèmes pour peu que la matrice ait un rang élevé ce qui est le cas du dépouillement de spectres. Rappelons que le rang de la matrice des moindres carrés défini en (i 6 ) est égal au nombre de constituants du spectre.

A l'inversion on préfère résoudre le système en y ajoutant des contraintes à savoir d'obtenir des solutions physiquement valables c'est-à-dire positives ou nulles.

Suivant l'algorithme de BEALE [6] on parvient à une telle résolution.

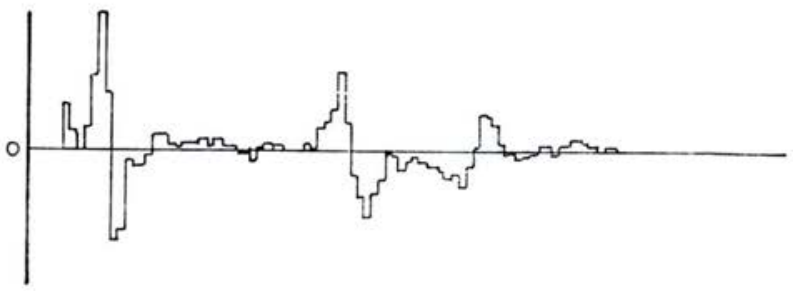

FIG. 15. - Tracé des résidus après dépouillement.

La figure is donne l'allure des résidus du dépouillement du spectre représenté par la figure I 3 , c'est-à-dire la valeur des erreurs statistiques de comptage. Une fois déterminées les proportions $X_{j}$ des étalons dans le mélange on recalcule le spectre complexe et on le déduit du spectre expérimental; à la vue de ce tracé on peut dire si le dépouillement est correct ou non.

\section{II.4 - ACTIVITÉ OU DOSE DU RAYONNEMENT POLYÉNERGÉTIQUE}

$X_{j}$ est la proportion d'étalons $j$ trouvée dans le spectre polyénergétique et $N_{j}$ le nombre de coups total du spectre $j$.

Le nombre de coups détectés par le scintillateur pour le rayonnement $j$ est donc $X_{j} N_{j}$.

Pour remonter à l'activité, si $\varepsilon_{j}$ est l'efficacité du détecteur

$$
A^{\prime}{ }_{j}=\varepsilon_{j} N_{j} x_{j} .
$$

$A^{\prime}{ }_{j}$ représente l'activité du flux atteignant le cristal. En multipliant par le facteur de géométrie $G$ et en tenant compte de l'absorption de la source $R_{j}$ il vient :

$$
A_{j}=\varepsilon_{j} \times N_{j} \times x_{j} \times G \times R_{j}(\text { en } \mathrm{Ci})
$$

Ceci étant fait pour chacun des spectres constituant le mélange, on peut ainsi remonter à la distribution énergétique de la source. 


\section{S - LES ANOMALIES DU DÉPOUILLEMENT}

L'application de la méthode décrite est quasi-infaillible quand on dispose de tous les spectres susceptibles d'entrer dans la composition de mélanges. Par contre l'allure des résidus de la figure 16 donne à penser qu'il a manqué un spectre lors du dépouillement, ce spectre ayant son pic d'absorption totale situé à $\nu$. Ceci peut se produire si pour constituer la spectrothèque l'élément a une vie trop courte pour être relevé ou que l'élément est trop coûteux.

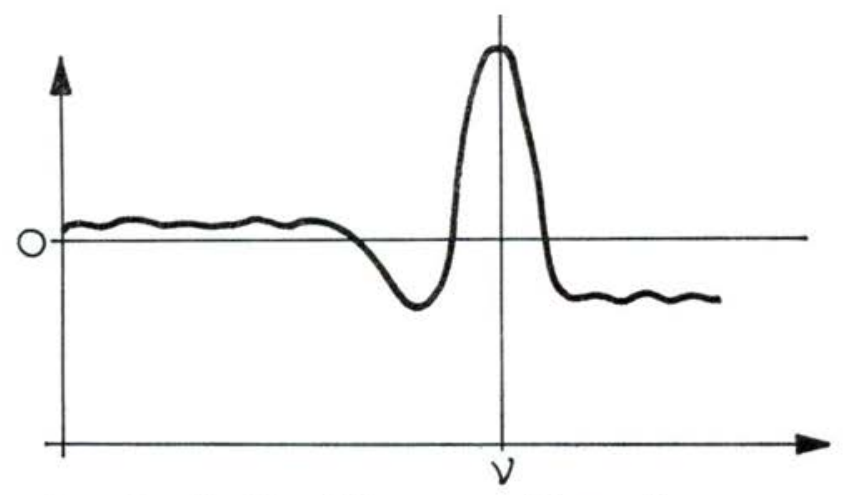

Fig. 16. - Tracé des résidus montrant l'absence d'un spectre.

Cette anomalie oblige à construire un spectre qu'on introduira dans la deuxième phase du dépouillement. Contrairement à ce qui a été exposé plus haut (I4), on n'utilise pas la méthode analytique d'interpolation sur des spectres voisins mais préférentiellement une méthode de Monte-Carlo reproduisant fidèlement les phénomènes physiques des interactions des gammas avec le détecteur $[7,8,9]$.

C'est donc un tel spectre qui va être inclus dans la spectrothèque utilisée pour le dépouillement et on a la possibilité par cette méthode de dépouiller au mieux les produits de fission frais.

La méthode de Monte-Carlo a l'avantage de donner une valeur à l'activité de l'émetteur responsable du spectre que l'on cherche à représenter. En effet, le nombre de photons tirés est fixé au départ et c'est lui qui exprime l'activité. L'allure du spectre obtenu est une forme semblable à la figure i i et en tenant compte de la propension des raies à l'étalement, la similitude d'un tel spectre avec un spectre relevé expérimentalement est grande, à la statistique de comptage près.

Pourtant pour appliquer cette méthode, une étude préliminaire de la réponse du détecteur est nécessaire. Tout d'abord la résolution au niveau du pic d'absorption totale : à partir du paramètre $b$ de la gaussienne ajustée (voir l'équation ro), il y a une relation entre celui-ci et l'énergie :

$$
b=C_{1} \sqrt{E}+C_{2} E[4]
$$

$C_{1}$ et $C_{2}$ sont les paramètres caractéristiques de la chaîne de détection. Pour une série de spectres témoins on relève la courbe $b=f(E)$ et on détermine $C_{1}$ et $C_{2}$. 
Ensuite la photofraction $F$ exprimant le rapport du nombre de coups sous le pic au nombre de coups dans le spectre entier donne une approximation du fond Compton à respecter. En jouant sur la géométrie du dispositif détecteur, cette photofraction et l'efficacité bien déterminées la construction de spectres par la méthode de M.C. ne pose aucun problème.

Pourquoi l'utiliser préférentiellement à l'interpolation analytique ? Bien que la méthode soit coûteuse elle a l'avantage de tenir compte de tous les effets et en particulier elle rend compte des pics de simple et double échappement pour des énergies élevées.

Et puis souvent les émissions $\gamma$ s'accompagnent d'émission $\beta$ détectables par le cristal et dont il faut tenir compte. Or comment alors interpoler? Enfin un spectre $\gamma$ n'est pas forcément monoénergétique et on doit tenir compte du diagramme de branchement.

\section{III - LA DÉTECTION DU RAYONNEMENT CONTINU}

\section{I - SPECTRE D'UN RAYONNEMENT CONTINU}

La diffusion d'un rayonnement gamma dans l'atmosphère, l'ambiance au voisinage d'un réacteur, la diffusion d'un rayonnement gamma dans un corps humain, donnent une idée d'un rayonnement continu détecté.

Après des collisions multiples l'énergie initiale des photons se dégrade et devient continue. Le problème revient donc à connaître la valeur du flux initial. Mais la dispersion de la chaîne de détection ne rend pas ce problème simple. Les figures 17,18 et 19 tentent d'expliquer le phénomène à partir d'une distribution continue d'énergie.

On ne peut résoudre effectivement l'équation - figure is - puis remonter au flux initial : par contre en discrétisant $F(V)$ de la façon suivante :

$$
F(V)=\sum_{h=0}^{n} \alpha_{h} f\left(E_{h}\right) K\left(V, E_{h}\right) \Delta E_{h}
$$

et l'information dans le canal $i$

$$
N_{i}=\int_{\Delta V_{i}} F(V) \mathrm{d} V=\sum_{h=0}^{n} \alpha_{h} f\left(E_{h}\right) \Delta E_{h} \int_{\Delta V_{i}} K\left(V, E_{h}\right) \mathrm{d} V
$$

\section{2 - LA RÉPONSE D'UN DÉTECTEUR A UNE BANDE D'ÉNERGIE}

Pour des raisons que nous avons vues plus haut nous nous refusons à exprimer $K\left(V, E_{h}\right)$ sous forme analytique. Il nous reste donc à calculer la réponse du détecteur pour toutes les énergies d'une manière presque continue. L'information expérimentale numérique étant donnée au niveau du canal, il paraît raisonnable de calculer la réponse pour chaque canal correspondant à un intervalle d'énergie $\Delta E_{h}$. 


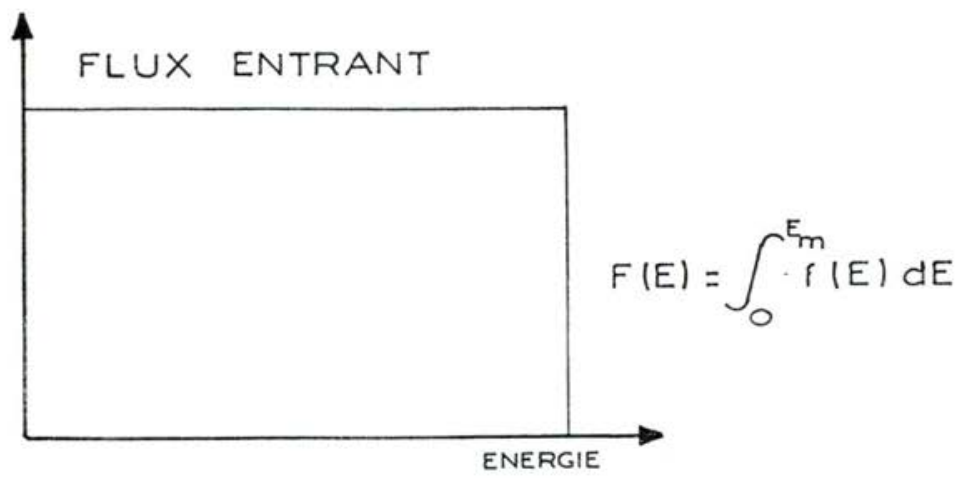

FIG. 17

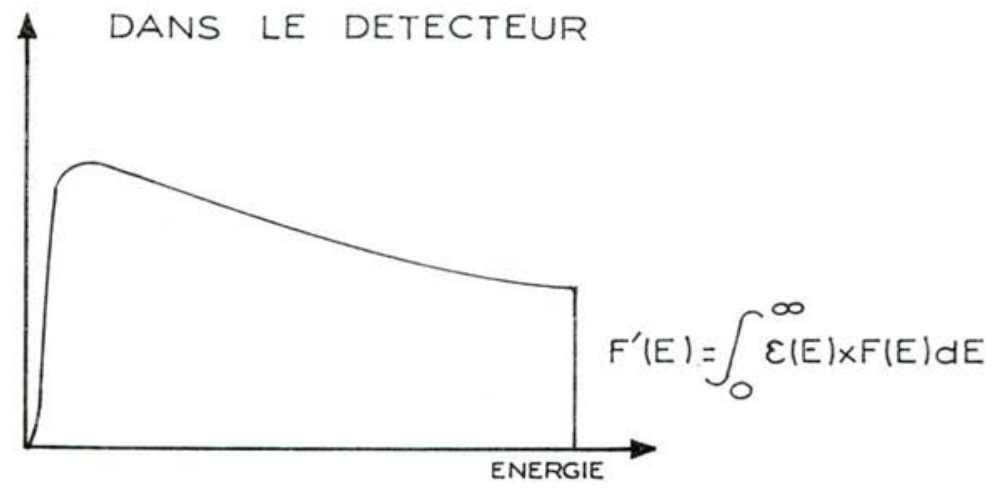

FIG. 18

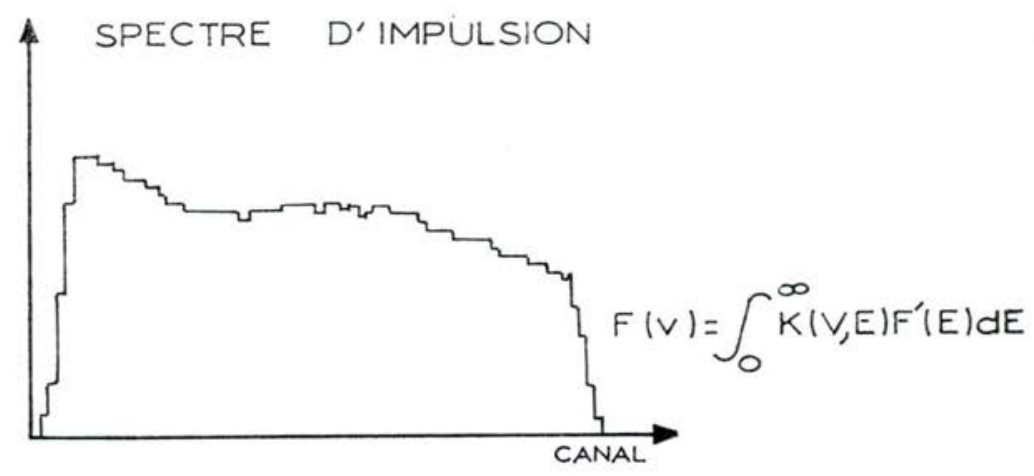

Fig. 19 
MESURE D'UN RAYONNEMENT GAMMA

37

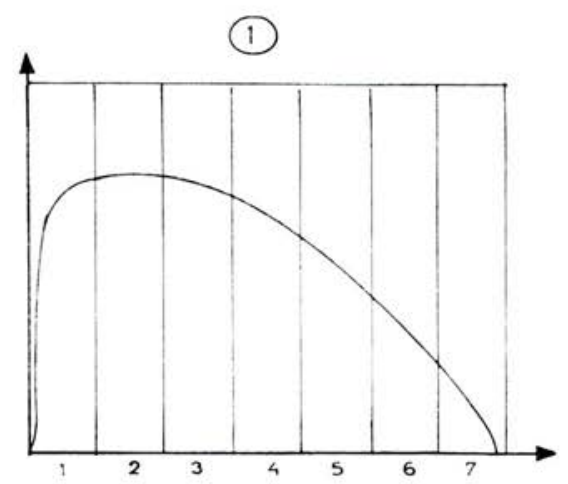

(2)
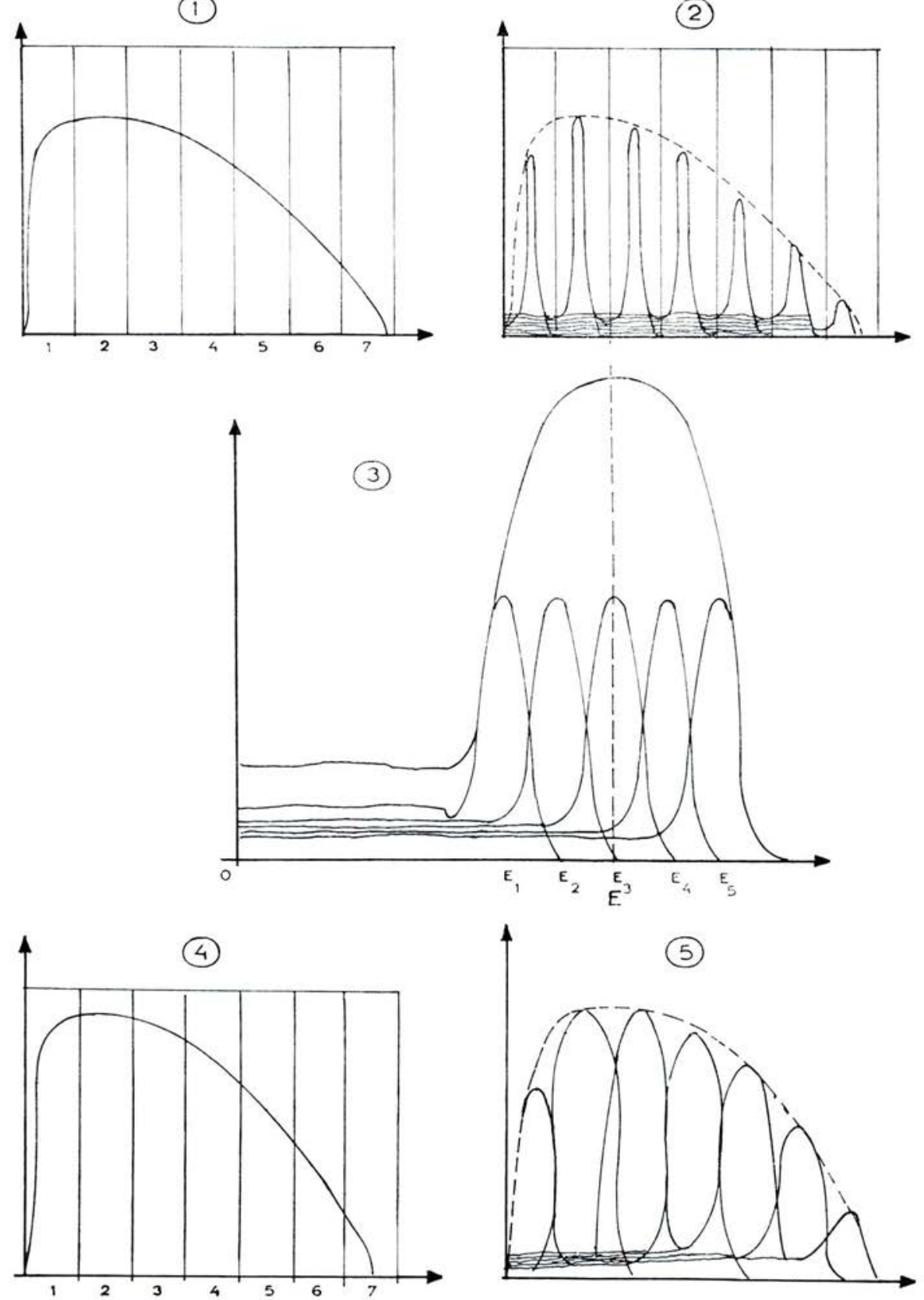

Fig. 20 
Cela conduirait naturellement à résoudre un système de 400 équations à 400 inconnues pour un sélecteur 400 canaux. Il en est hors de question.

Il s'agit donc de faire des partitions :

I) soit en construisant un spectre réponse moyen pour une partition,

2) soit en construisant un spectre de bande réponse à une gamme finie d'énergies.

I er cas: Dans la figure 20 (1) représente un spectre continu qu'on a partagé en 7 zones. (2) est l'approximation que l'on fait en disant que le spectre continu est combinaison linéaire de spectres réponses construits à partir de l'énergie moyenne de la zone.

On voit tout de suite qu'un tel dépouillement sera très mauvais, la matriceréponse étant presque vide. La forme des résidus témoignera du manque d'informations.

$2^{\mathrm{e}}$ cas : Reprenant le découpage en zones, on établit, (3), un spectre de bande somme de spectres élémentaires construits eux à partir de l'énergie moyenne correspondant aux milieux des canaux. Ici on a figuré un spectre composé de 7 spectres séparés, c'est-à-dire un spectre de bande sur 7 canaux.

En (5) est tracé le spectre continu et les spectres-bandes servant au dépouillement. La vacuité observée dans le premier cas n'existe plus et le dépouillement sera bon.

Tous les spectres de bande sont bien entendu construits par la méthode de Monte-Carlo; il aurait été impossible de les construire autrement.

Comme exemple concret, la figure 2 I illustre un spectre continu et son dépouillement. Il a été partagé en I I zones d'inégale largeur $i$.

et

5 bandes de roo keV de largeur,

3 bandes de $200 \mathrm{keV}$ de largeur,

3 bandes de $300 \mathrm{keV}$ de largeur.

Chaque bande est somme des spectres réponses monoénergétiques pris de ro en $10 \mathrm{keV}$ pour la première bande: $5,15,25,35,45,55,65,75,85$ et $95 \mathrm{keV}$ l'énergie moyenne de la bande est égale à $50 \mathrm{keV}$.

Si les bandes sont inégales c'est parce que l'on désire connaître au mieux l'activité dans la zone des basses énergies, mais le procédé est très maniable et l'on peut dépouiller avec finesse telle ou telle partie d'un spectre.

III. 3 - LE DÉPouillement ET LES RÉSUltats

L'activité de chaque spectre de bande est déterminée par le nombre de photons qui ont servi à construire ce spectre. La formule de dépouillement est strictement la même que dans le cas d'un spectre discret.

$$
b_{i}=\sum_{j} a_{i j} x_{j}+Z_{i}
$$


$a_{i j}$ est le nombre de coups dans le canal $i$ du spectre de bande $j$,

$x_{j}$ est la proportion du spectre de bande $j$ dans le spectre continu, et $Z_{i}$ l'erreur statistique.

On ne constate pas lors d'un dépouillement de discontinuité des résidus dus à un décalage ou à un spectre manquant.

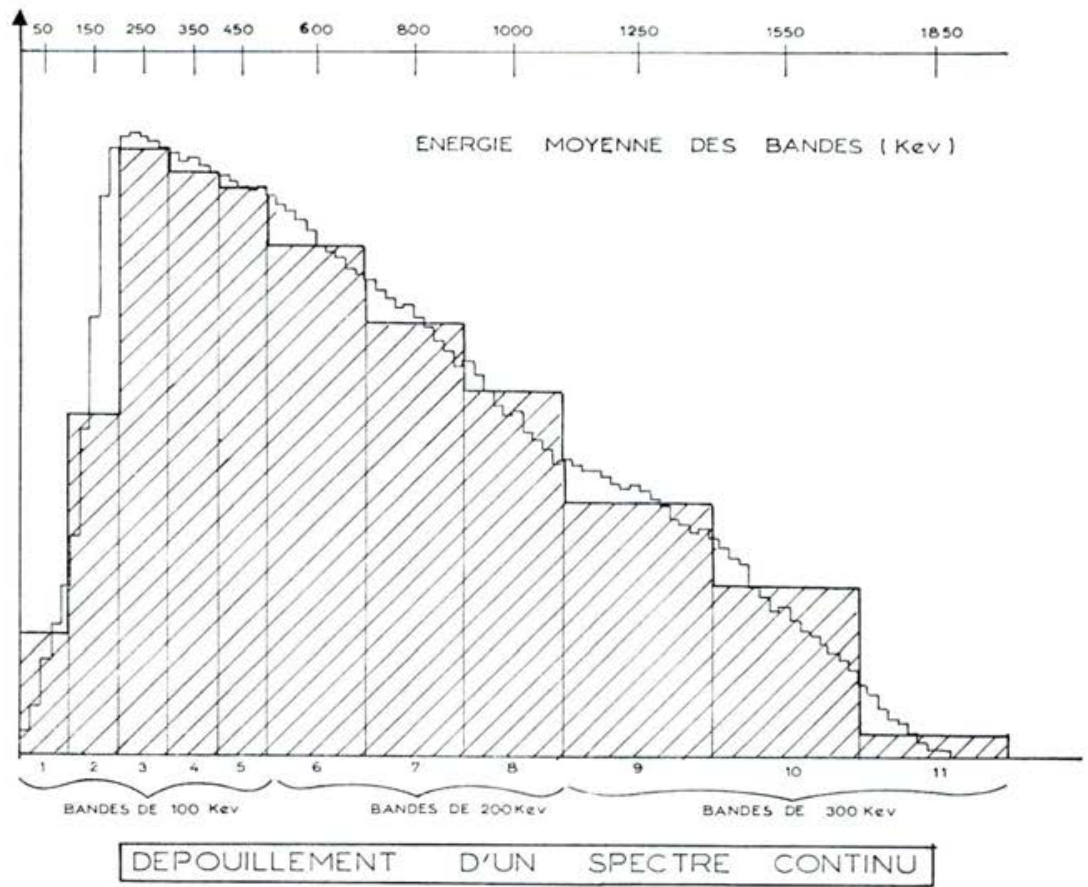

FIG. 21

Le tableau II donne les résultats d'un dépouillement du spectre continu.

Le dépouillement a été fait à partir du $50^{e}$ canal et à l'aide de 30 spectres de bande sur 400 canaux.

On a défini l'énergie moyenne de chaque bande; on lui attache une efficacité moyenne $\bar{\varepsilon}_{j}$ et le nombre de photons détectés $N_{j}$. L'activité d'une tranche d'énergie s'écrit alors :

$$
A_{j}=x_{j} \times \bar{\varepsilon}_{j} \times N_{j} \times G \times \bar{R}_{j}
$$

$G$ facteur de géométrie

et $\overline{R_{j}}$ coefficient d'absorption moyen de la source - valant généralement I quand on calcule seulement le flux de rayonnement entrant dans la chaîne de détection. 
TABLEAU II

\begin{tabular}{|c|c|c|c|}
\hline \multicolumn{2}{|c|}{$\begin{array}{l}\text { SPECTRE } \\
\text { ÉTALON }\end{array}$} & NUMÉRO & \multirow{2}{*}{$\begin{array}{c}\text { PROPORTION } \\
. .00\end{array}$} \\
\hline 5 & 95 M.C. & *DAM $\quad \mathrm{I} *$ & \\
\hline 105 & I9s M.C. & *DAM $\quad 2^{*}$ & .000 \\
\hline 205 & 295 M.C. & *DAM $3 *$ & .000 \\
\hline 305 & 395 M.C. & *DAM 4* & .000 \\
\hline 405 & 495 M.C. & *DAM $5 *$ & .000 \\
\hline 505 & 595 M.C. & *DAM $6 *$ & .000 \\
\hline 605 & 695 M.C. & ${ }^{*} \mathrm{DAM} \quad 7^{*}$ & .000 \\
\hline 705 & 795 M.C. & *DAM $\quad 8^{*}$ & .610 \\
\hline 805 & 895 M.C. & *DAM $\quad 9^{*}$ & .601 \\
\hline 905 & 995 M.C. & *DAM ro* & .408 \\
\hline 1005 & I095 M.C. & *DAM $\mathrm{II}^{*}$ & .347 \\
\hline Iros & II95 M.C. & *DAM $12 *$ & .321 \\
\hline 1205 & I295 M.C. & *DAM $13 *$ & .292 \\
\hline 1305 & 1395 M.C. & *DAM I $4^{*}$ & .282 \\
\hline 1405 & I495 M.C. & *DAM Is* & .282 \\
\hline Isos & Is95 M.C. & *DAM $16^{*}$ & .280 \\
\hline 1605 & 1695 M.C. & *DAM $17 *$ & .296 \\
\hline 1705 & I795 M.C. & ${ }^{*} \mathrm{DAM}$ r $8 *$ & .252 \\
\hline 1805 & 1895 M.C. & *DAM 19 * & .227 \\
\hline 1905 & I995 M.C. & *DAM 20* & .225 \\
\hline 2005 & 2245 M.C. & *DAM $28 *$ & .180 \\
\hline 2255 & 2495 M.C. & *DAM $2 \mathrm{I}^{*}$ & .116 \\
\hline 2505 & 2745 M.C. & *DAM $22^{*}$ & .083 \\
\hline 2755 & 2995 M.C. & ${ }^{*} \mathrm{DAM} \quad 23^{*}$ & .060 \\
\hline 3005 & 3245 M.C. & *DAM $24^{*}$ & .042 \\
\hline 3255 & 3495 M.C. & ${ }^{*}$ DAM $25 *$ & .029 \\
\hline 3505 & 3745 M.C. & *DAM $26 *$ & .020 \\
\hline 3755 & 3995 M.C. & *DAM $27^{*}$ & .018 \\
\hline 4005 & 4995 M.C. & *DAM $29^{*}$ & $.06 \mathrm{I}$ \\
\hline 5005 & 5995 M.C. & *DAM 30* & .007 \\
\hline
\end{tabular}

Dans le cas de réaction d'activation, il se produit un spectre continu sur lequel se superposent des pics correspondant à des émissions monoénergétiques. Là il est nécessaire, en plus du fond continu d'adjoindre à la spectrothèque les spectres caractéristiques de ces rayonnements. L'opération nécessite beaucoup de soins car le calage de ces derniers spectres est impératif.

\section{CONCLUSION}

La mesure de la dose de rayonnement ou de l'activité des sources donne de précieux renseignements en radioprotection. Nous venons de voir l'enchaînement des opérations pour atteindre la valeur d'un flux de rayonnement; cela impose de connaître parfaitement l'appareil détecteur utilisé. En traitant le problème dans les deux sens - du calcul théorique du flux à la sortie de l'appareil de mesure au calcul à partir des données expérimentales du flux analysé - pour une chaîne de détection donnée, on arrive à saisir tous les phénomènes intervenant dans la détection. 
Plusieurs programmes ont été mis au point au L.C.A. Ecrits pour UNIVAC I 108 et I.B.M. 7094 - ils répondent dans la majeure partie des cas aux problèmes à traiter. Néanmoins signalons que c'est grâce à ces puissants ordinateurs que le traitement des informations numériques issues d'un sélecteur multicanaux est rendu possible, surtout que ces sélecteurs arrivent à totaliser 4096 canaux.

\section{PROGRAMME DE SPECTROMÉTRIE GAMMA}

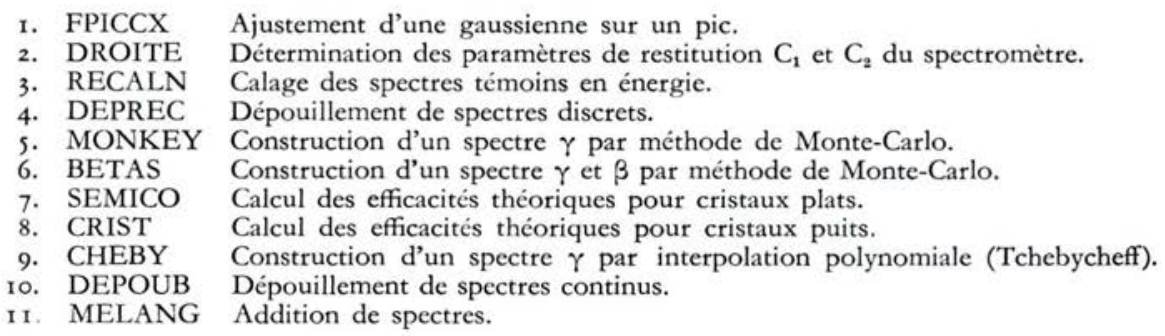

\section{RÉFÉRENCES}

(1) Bonnet C., Hillion P., Nurdin G. - Nucl. Inst. and Meth. 54 (1967), 321.

(2) SNyder J. - Nucleonics, Fev. 65, Vol. 23, no 2. - Verheijke. - Inter. J. Applied Rad. Isot. 1964: Vol. Is, 559-563.

(3) DAM/DTAT/LCA. - Journée d'Etudes sur le Traitement Numérique des Spectres Gamma, février 1967.

(4) Heath R.L., T.I.D., 7594 - Proceeding of the total absorption Gamma Ray Spectrometry Symposium, May 10-1 1-60, p. I6.

(5) Chester R.O., T.I.D., 7594. - Op. cit.

(6) Beale E.M.L. - «On quadratic Programming ", Naval Res. Logistics, Quant 6 (sept. 1959).

(7) Miller W.F., Snow W.J. ANL 6318 - T.I.D., 4500. - Monte-Carlo Calculation of the energy loss spectra for Gamma Rays in Sodium Iodide.

(8) Zerby C.D., Mayer A., Munay R.B. - Nuclear Instr. and Methods I 2 (1961), p. I15-123.

(9) DMA/DTAT/LCA. - Journée d'Etudes sur le Traitement Numérique des Spectres Gamma, février 1967. 